\title{
INF2 mutations often implicated in autosomal dominant focal segmental glomerulosclerosis
}

$\mathrm{M}$ utations in the INF2 gene, which encodes a member of the

formin family of actin-regulating proteins, cause a high proportion of cases of autosomal dominant focal segmental glomerulosclerosis (FSGS), according to a study published in the Journal of the American Society of Nephrology.

Most cases of inherited FSGS occurring in childhood display an autosomal recessive type of inheritance. However, ACTN4 and TRPC6 genes have been shown to be implicated in the rare autosomal dominant forms of the disease occurring in juveniles and adults, accounting for about $4 \%$ and $6 \%$ of familial FSGS, respectively.

An article by Elizabeth Brown and colleagues published in Nature Genetics in 2010 reported the identification of nine independent missense mutations in INF2 that were found to segregate with autosomal dominant FSGS in 11 unrelated families. These researchers hypothesized that individuals with disease-associated INF2 mutations have a defect in actin-mediated podocyte structural maintenance and repair.

As Brown et al.'s finding had not been confirmed by other studies, and because the role of INF2 mutations in sporadic cases of FSGS was unknown, Olivia Boyer et al. decided to look further into the prevalence of INF2 mutations in FSGS. These researchers screened 54 families (78 patients) with a glomerular proteinuric disorder that seemed to show an autosomal dominant mode of inheritance. Mutations in ACTN4 and TRPC6 were excluded in all cases.

"Given the occurrence of incomplete penetrance in autosomal dominant FSGS cases and the reports of neomutation in ACTN4-, TRPC6- and INF2-related FSGS, we also searched for INF2 mutations in 84 sporadic cases of FSGS," notes Boyer. No differences were observed between familial and sporadic cases in

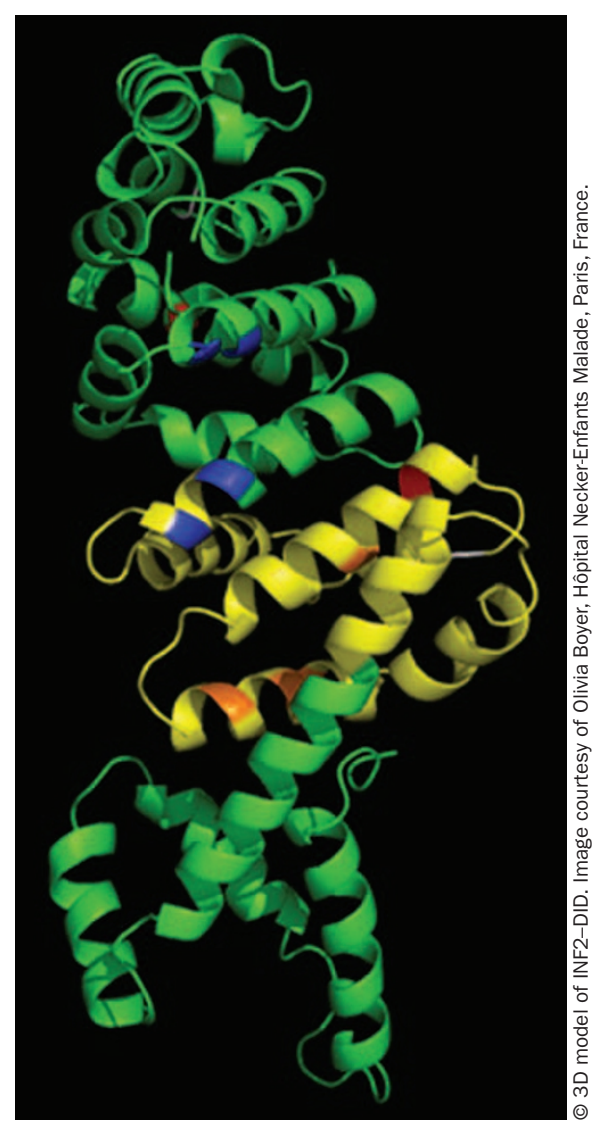

terms of ethnicity, histology or clinical presentation.

Among familial cases, proteinuria occurred at median age of 20.5 years (range 2-52 years), and FSGS was documented in at least one affected family member in $80 \%$ of families. The researchers found seven missense INF2 mutations in nine families (28 patients), a detection rate of $16.7 \%$. Median age at proteinuria onset was higher in patients with INF2 mutations than in those without (27 years versus 15.5 years; $P=0.026$ ).

Only one patient with sporadic FSGS (who presented with proteinuria and FSGS lesions at 15 years of age) was found to have a known INF2 mutation (p.R218Q). Complete data from the patient's apparently healthy parents were not available to determine whether this patient had a de novo mutation or whether this patient was a familial case with low penetrance and variable expressivity.

Boyer et al. found that all variants of the INF2 mutation were localized in exons 2 and 4 and caused nonconservative changes in highly conserved amino acids of the N-terminal diaphanous inhibitory domain (DID) of INF2, a region that interacts with the $\mathrm{C}$-terminal diaphanous autoregulatory domain (DAD), competing for actin monomer binding and inhibiting depolymerization. Six of the seven distinct altered residues were found to localize to an INF2 region corresponding to an mDia1 DID subdomain that is reported to co-immunoprecipitate with IQ motifcontaining GTPase-activating protein (IQGAP1), which is known to interact with the podocyte proteins nephrin and PLCE1.

The results of this study confirm that INF2 mutations are responsible for a high proportion of autosomal dominant cases of FSGS, greater than the proportion of cases caused by mutations in ACTN4 or TRPC6. The authors recommend that screening for INF2 mutations, at least in exons 2 to 4, should be strongly considered in patients with an autosomal dominant history of FSGS. "Although INF2 is the major known gene associated with autosomal dominant FSGS, it explains only $12-17 \%$ of cases, suggesting the implication of other podocyte genes, yet to be identified," notes Boyer. "Therefore, our group continues to look for new genes involved in familial proteinuric disorders."

\section{Rebecca Ireland}

\footnotetext{
Original article Boyer, 0. et al. Mutations in INF2 are a major cause of autosomal dominant focal segmental glomerulosclerosis. J. Am. Soc. Nephrol. 22, 239-245 (2011)

Further reading Brown, E. J. et al. Mutations in the formin gene INF2 cause focal segmental glomerulosclerosis. Nat. Genet. 42, 72-76 (2010)
} 